\title{
A new species of titi monkey, Callicebus Thomas, from north-eastern Brazil (Primates, Cebidae)
}

\author{
Shuji Kobayashi ${ }^{1}$ \\ Alfredo Langguth ${ }^{2}$
}

\begin{abstract}
A new species of titi monkey, Callicebus, is described based on five specimens obtained from the state of Sergipe, north-eastern Brazil. It is a member of the Personatus group, but is easily distinguished from the other forms. The most noticeable differences are in the pelage coloration, the forehead, crown, and ear being black, and having a zebra-like, striped pattern on the anterior half of the back. Distinct features of its dental morphology and craniometry are also described.

KEY WORDS. Callicebus, Cebidae, new species, Personatus group, north-eastern Brazil
\end{abstract}

The genus Callicebus Thomas, 1903 (Cebidae) occurs in forested areas of tropical South America, in the Amazon and the Atlantic forest of Brazil. The most recent taxonomic revision of the titi monkeys was carried out by HERSHKOVITZ (1990) who recognised 13 species and 17 species and subspecies, although his arrangement, has not received widespread acceptance by other researchers (e.g. REDFORD \& EISENBERG 1992). KOBAYASHI (1995) classified the forms of Callicebus into five phyletic species groups: Donacophilus, Cupreus, Moloch, Torquatus and Personatus. In this paper the group names are in the sense of this author. The Personatus group includes four different forms (personatus E. Geoffroy, 1812, nigrifrons Spix, 1823, barbarabrownae Hershkovitz 1990, and melanochir WiedNeuwied, 1820) which occur in the Atlantic forest in eastern and south-eastern Brazil (Fig. 3). The titi monkeys comprising the Personatus group are classified as subspecies by HERSHKOVITZ $(1988,1990)$ but here it is argued that they should be considered distinct species (see Discussion), and refer to them as such in this article.

During an expedition in 1994 in the state of Sergipe, north-eastern Brazil, two dead titi-monkeys were obtained from a native hunter in the Fazenda Santana. These showed a pelage coloration quite different from that of the four forms of personatus recognised at the time, and, on further examination, some distinct features of their dental morphology were also found. Two more specimens were obtained in 1995, in the hands of another native hunter at the Fazenda Arauari, Sergipe, and later a fifth specimen was studied in the collection of the Museu

1)Department of Asian Studies, Chukyo Women's University. Nadakayama 55, Yokone-cho, Aichi 474-0011, Japan.

2) Departamento de Sistemática e Ecologia, Universidade Federal da Paraíba. 58059-900 João Pessoa, Paraíba, Brasil. 
Nacional, Rio de Janeiro, collected at the Fazenda Cruzeiro, Sergipe. Multivariate analysis revealed that the craniometric differences of this titi were of a degree which corresponded to those found in other forms of Callicebus ranked as species in the other phyletic groups of KOBAYASHI (1995). This new species is described below.

\section{MATERIAL AND METHODS}

The five specimens of the new species were all female, no males are available. They were compared directly with the forms of the Personatus ( $C$. personatus, C. nigrifrons, C. melanochir and C. barbarabrownae) and Moloch $(C$. brunneus Wagner, 1842, C. hoffmannsi hoffmannsi Thomas, 1908, C. h. baptista Lönnberg, 1939, C. moloch Hoffmannsegg, 1807 and C. cinerascens Spix, 1823), groups following KOBAYASHI (1995). Only a skin was available for C. barbarabrownae. Comparisons were also made with other species based on descriptions from the literature.

The following external measurements were taken: body weight (BW), head and body length $(\mathrm{H} \& \mathrm{~B})$, tail length $(\mathrm{T})$, hind foot length, with and without claw (HF), and ear length (E). Non-metric characters of dental morphology used for comparison included: the position of cusps on the upper second incisor $\left(\mathrm{I}^{2}\right)$; the morphology of the metaloph on the upper first $\left(\mathrm{M}^{1}\right)$ and second $\left(\mathrm{M}^{2}\right)$ molars; the frequency of appearance of the paraconule on $\mathrm{M}^{1}$ and $\mathrm{M}^{2}$; and the angle of the cristid obliqua on the lower first $\left(\mathrm{M}_{1}\right)$ and second $\left(\mathrm{M}_{2}\right)$ molars. Skull measurements used in multivariate analyses are shown in figure 1, measuring points followed MARTIN \& SALLER (1957) and Kobayashi (1995). However, in figure 1 of KoBAYASHI (1995) measurements Nos. 5 and 22 were replaced by Nos. 6 and 23 and vice versa. These 23 linear measurements were taken with a digital caliper $(15 \mathrm{~cm}$ NSK MAX-cal model), and were recorded to the nearest $1 / 100 \mathrm{~mm}$. The data set for each form was comprised of combined measurements of males and females because there are no appreciable differences between the sexes at comparable ages (KOBAYASHI 1995). Two analytical procedures, distance analysis by Q-mode correlation coefficients and principal component analysis, were applied in order to examine the degree of cranial differentiation. Clustering, using the furthest neighbours method was performed for the distance matrix, converted from the similarity matrix of the Q-mode correlation coefficients. Clustering is one of the most powerful methods for determining morphometric distances among various forms without loss of information (SNEATH \& SOKAL 1973). After clustering, ordination by the quantification theory model 4 was employed for the distance matrix, valuable for understanding the data structure in more detail (SNEATH \& SoKAL 1973). Pooled correlation coefficients of the variables were used in the principal component analysis. Specimens with missing measurements could not be used because this statistical technique requires a complete data set. Three-dimensional scatter diagrams were drawn based on the mean scores of the first, second, and third principal components. The analyses were carried out after confirmation of probable normality and homoscedasticity in C. moloch and C. personatus (these two forms were chosen as representatives due to their relatively good sample sizes). 

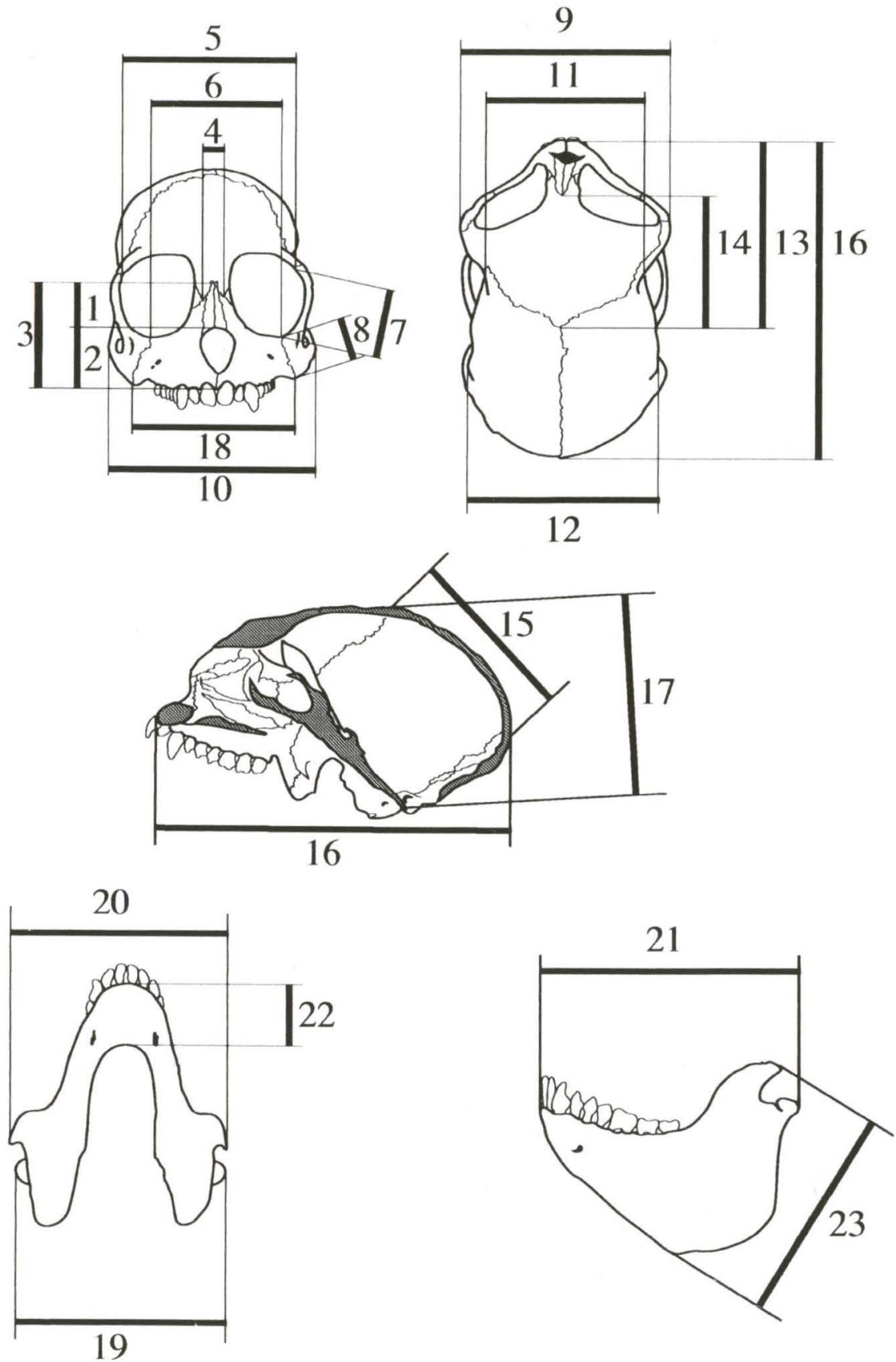

Fig. 1. Skull measurements used in this study. For the definition of the measuring points see KOBAYASHI (1995). 
The specimens examined are deposited in the following mammal collections: Museu Nacional, Rio de Janeiro (MN); Departamento de Sistemática e Ecologia, Universidade Federal da Paraíba, João Pessoa (UFPB); Museu de Zoologia, Universidade de São Paulo, São Paulo (MZUSP); National Museum of Natural History, Washington D.C. (USNM); American Museum of Natural History, New York (AMNH); Field Museum of Natural History, Chicago (FMNH); Departamento de Zoologia, Universidade de Brasília (UNB).

\section{Callicebus coimbrai sp.n.}

Figs 3-6

\section{Holotype}

UFPB 1599 (Original No. AL 3516). Adult female, stuffed skin, complete skeleton. Nasal, right side of parietal, temporal, occipital, zygomatic, sphenoid bones and coronoid process slightly damaged. Obtained from a native hunter by Shuji Kobayashi and Alfredo Langguth on January 30, 1994.

Type-locality. Proximity of the small village Aragão, in the region of Santana dos Frades about $11.0 \mathrm{~km} \mathrm{SW}$ of Pacatuba, state of Sergipe, Brazil (GPS 10²'S, $36^{\circ} 41^{\prime} \mathrm{W}$, alt. $90 \mathrm{~m}$ ). The locality is south of the estuary of the Rio São Francisco.

\section{Paratypes}

UFPB 1600 (Orig. No. AL 3517). Young adult female, stuffed skin, complete skeleton frontal and both parietal bones damaged; same locality and date as UFPB 1599. This specimen will be deposited in the Natural History Museum of Kyoto University, Japan. UFPB 1859 (Orig. No. AL 3586), adult female, stuffed skin, complete skeleton, obtained from a native hunter by Shuji Kobayashi and Alfredo Langguth on January 23, 1995 at Fazenda Arauari, about $10 \mathrm{~km}$ ESE of Maruim, some large forest patches surrounding a transmission tower of the Brazilian Communications Company - Embratel - on the road to Porto de Sergipe, state of Sergipe, Brazil (GPS $10^{\circ} 45^{\prime} \mathrm{S}, 37^{\circ} 00^{\prime} \mathrm{W}$, alt. $75 \mathrm{~m}$ ). UFPB 1860 (Orig. No. AL 3587), adult female, stuffed skin, complete skeleton, right nasal, frontal, left lachrymal, left ethmoid, maxillas and right sphenetmoid bones slightly damaged; same locality and date as UFPB 1859. MN 30550 (Orig. No. AL 3078), adult female, stuffed skin, complete skeleton, obtained from a native hunter during field work of the project "Inventário Faunístico da Mata Atlântica" (1992-1995) co-ordinated by the Fundação Biodiversitas (Belo Horizonte), and financed by the John D. and Catherine T. MacArthur Foundation (Chicago), at Fazenda Cruzeiro, about $13 \mathrm{~km}$ SSE of Cristinápolis, on the left margin of the Rio Real, state of Sergipe, Brazil (about $11^{\circ} 29^{\prime} \mathrm{S}, 37^{\circ} 46^{\prime} \mathrm{W}$, alt. c. $100 \mathrm{~m}$ ).

\section{Geographic distribution}

This new titi monkey is known from the three localities listed above, between the Rio São Francisco and the Rio Real which marks the southern border of the state of Sergipe. It is probably restricted to the south of the Rio São Francisco, but the southern limit to its range is unknown. Titis have been reported near the right bank of the Rio Real in the south. OLIVER \& SANTOS (1991), for example, obtained reports 
of titi monkeys from the vicinities of Cachoeira do Abadia and Jandaira, on the south bank of the Rio Real, in the north-east of the state of Bahia. The western limit of the distribution is also unknown. C. coimbrai is a true inhabitant of the Atlantic coastal forest, and it is believed that its range does not extend west to the highlands inland where the vegetation is drier and characterised by caatinga - thorn scrub, deciduous forest and gallery forest (RIzZINI et al. 1988). Titis occurring there are probably referable to the form barbarabrownae (Fig. 2).

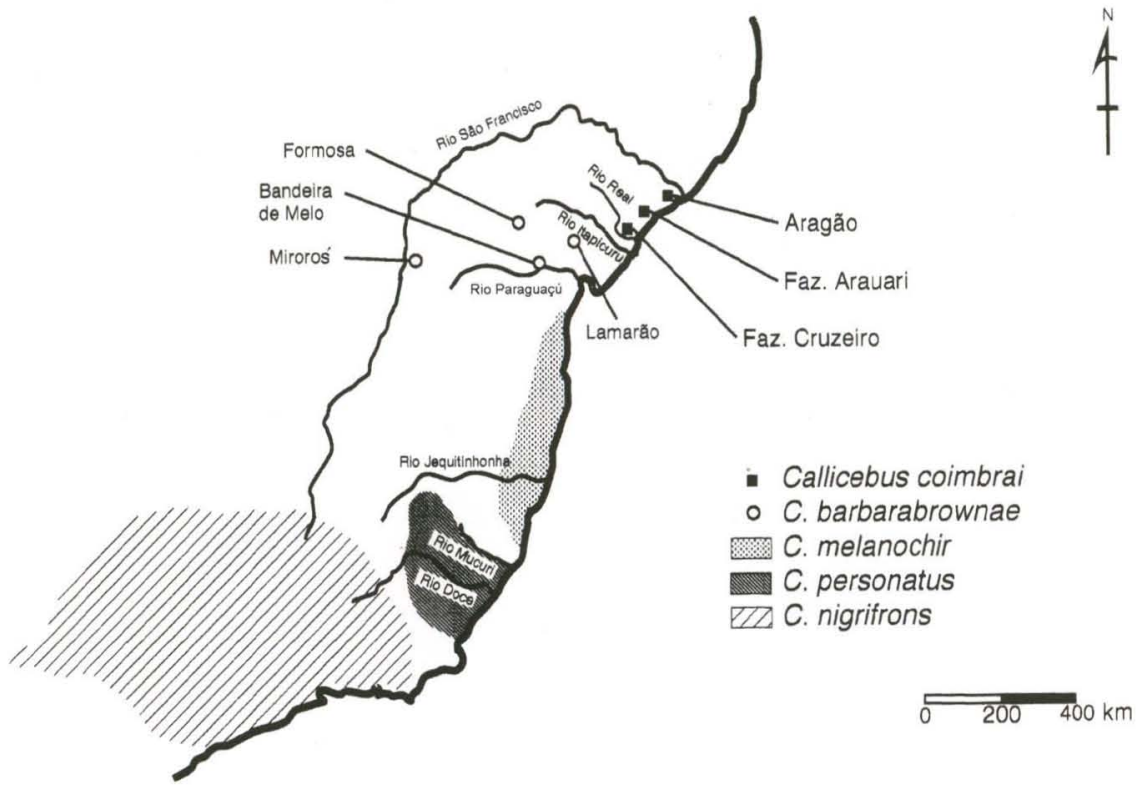

Fig. 2. Geographic distribution of members of the Callicebus personatus group, following KINZEY (1982), HERSHKOVITZ (1990) and this study.

\section{Habitat}

In common with other masked titis, C. coimbrai is apparently abundant in the dense understorey and lower canopy.

\section{Diagnosis}

A titi of the Personatus group (sensu KoBAYASHI 1995), but quite easy to distinguish from the other members of the group by the black forehead, crown and ear and the buffy trunk. The metaloph on the upper first and second molars is fragmented, and takes the form of two conical conules instead of a continuous crest.

\section{Etymology}

The species is named in honour of Adelmar F. Coimbra-Filho, founder and ex-Director of the Rio de Janeiro Primate Centre (CPRJ/FEEMA), in recognition of his research and valuable contributions to the biology and conservation of Brazilian primates. 

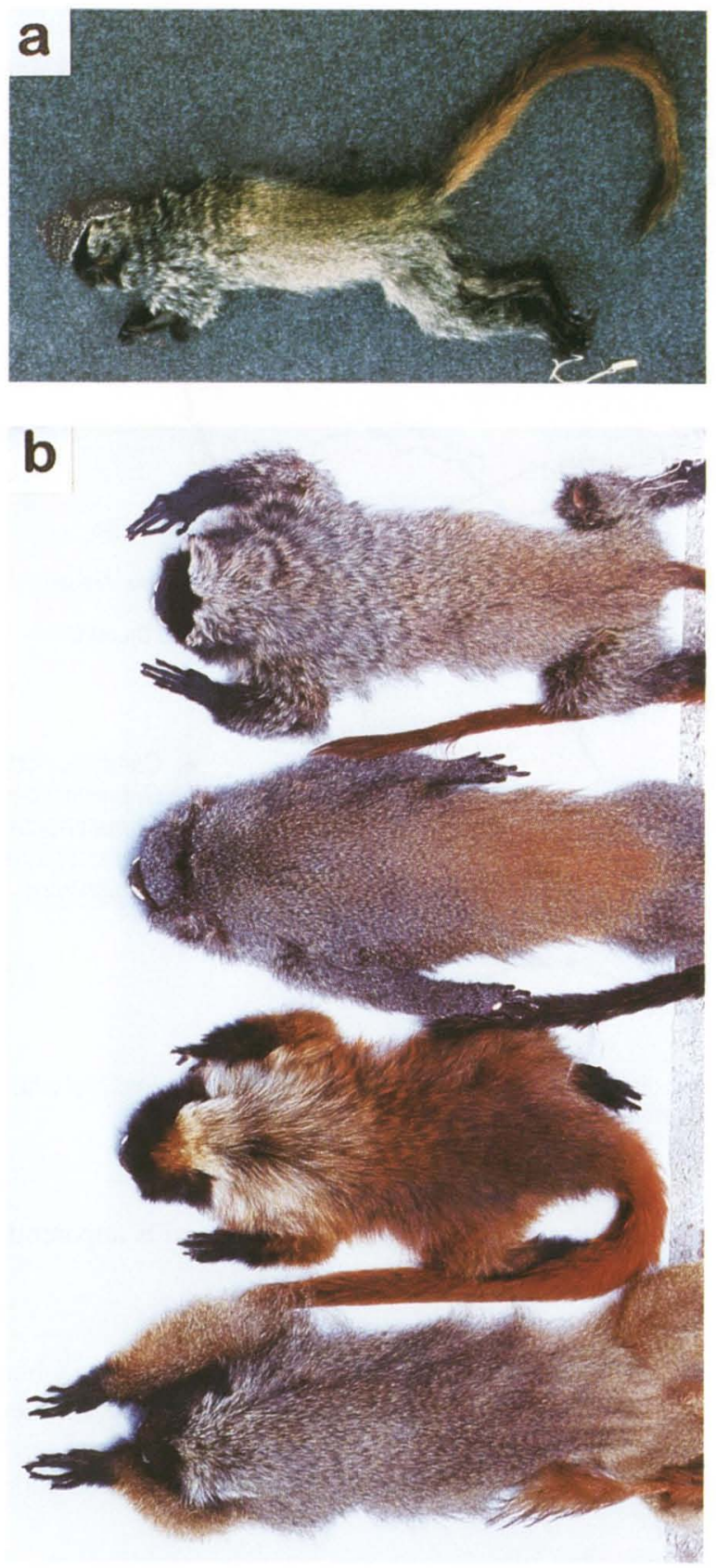

Fig. 3. (A) Fresh specimen of $C$. coimbrai, holotype; (B) dorsal view of stuffed skins, from top to bottom: Callicebus coimbrai holotype, Callicebus melanochir (UFPB 2217), Callicebus personatus (UFPB 412), Callicebus nigrifrons (UFPB 2224).

Revta bras. Zool. 16 (2): 531 - 551, 1999 


\section{Description of the holotype}

Pelage. Forehead, crown, and ear black (a few buffy hairs on the forehead) and well demarcated from the other parts of the body; hairs on posterior crown whitish, the fine tips blackish, bases dark; nape also whitish, the fine tips of hairs blackish, bases dark (eumelanin) (Figs 3, 4b), cheeks dominantly blackish, but with some hair tips buffy, remainder of shaft black; the side of the cheek has a buffy vertical stripe (Fig. 4a); shoulders, upper arms, forearms, thighs and legs buffy, the fine tips of hairs blackish with subterminal pheomelanin band followed by a eumelanin band and another pheomelanin band, hair bases eumelanin; anterior back of trunk buffy with faint black zebra-like stripes (Fig. 3), hair band pattern same as the sides of the trunk; sides of trunk paler buffy, the hair tips blackish with three oheomelanin bands, hair bases eumelanin; posterior part of trunk brownish buffy, banding pattern of hairs same as the shoulders but some of the pheomelanin bands more pigmented (Figs 3, 4b); chest, throat and belly paler buffy, the fine tip of the hairs blackish with two pheomelanin bands, hair bases eumelanin; hands, wrists, ankles and feet blackish; tail dominantly orange, the hairs orange in outer half and brown in inner half; all underfur eumelanin coloured; skin of face, ear, palm and sole blackish.

Skull. Skull morphology is similar in general appearance to that of other members of the Personatus group (Fig. 5); overall size is comparatively small; pterygoid processes vestigial; parapterygoid processes rectangular and well developed; frontal bone behind supraorbital ridges depressed; frontal sinus in glabella comparatively little pneumatized; interorbital septum wide, not perforated; infraorbital fissure anteroposteriorly long; mesopterygoid fossa more nearly $\Omega$-shaped than U-shaped; small caudal projections present on the free border near the sagittal suture of palatines; lower part of temporozygomatic suture ventrally projected; bullae also ventrally projected; two infraorbital foramina on left and one on right sides; mandibular height lower; coronoid process elongate, hook-like and extends well above the condylar process. Measurements are shown in table I.

Teeth. All teeth considerably worn; tooth rows are similar to those of the other members of the Personatus group, more nearly V-shaped than U-shaped (Figs $5,6 \mathrm{~d})$; diastema between $\mathrm{I}^{2}$ and upper canine very small; no diastema in lower tooth row; $I^{1}$ with conule on lingual cingulum; cusp of $\mathrm{I}^{2}$ located distally (Fig. 6a); buccal cingulum on second premolar $\left(\mathrm{P}^{2}\right)$ vestigial but present on mesial and distal extremity, lingual cingulum worn and not recognisable; buccal and lingual cingula on third $\left(\mathrm{P}^{3}\right)$ and fourth $\left(\mathrm{P}^{4}\right)$ premolars present; on $\mathrm{M}^{1}$ stylar cusps on buccal cingulum present, metaloph dented in distobuccal, middle and mesiolingual parts, paraconule (sensu KOBAYASHI 1995) present, distal extremity of lingual cingulum connected to hypocone extending around its distolingual slope (same as type 1 of KOBAYASHI 1990), prehypocrista not connected to the metaloph but to the postprotocrista; on $\mathrm{M}^{2}$ one stylar cusp present, metaloph and lingual cingulum same as $\mathrm{M}^{1}$; cristid obliqua on $\mathrm{M}_{1}$ and $\mathrm{M}_{2}$ shapely curved inward (Fig. 6a,c).

\section{Variation}

Pelage. In UFPB Nos. 1859 and 1860 the whitish cheek bands are wider, and the zebra-like pattern of anterior back is rather less distinct (Figs 3, 4b); in MN 

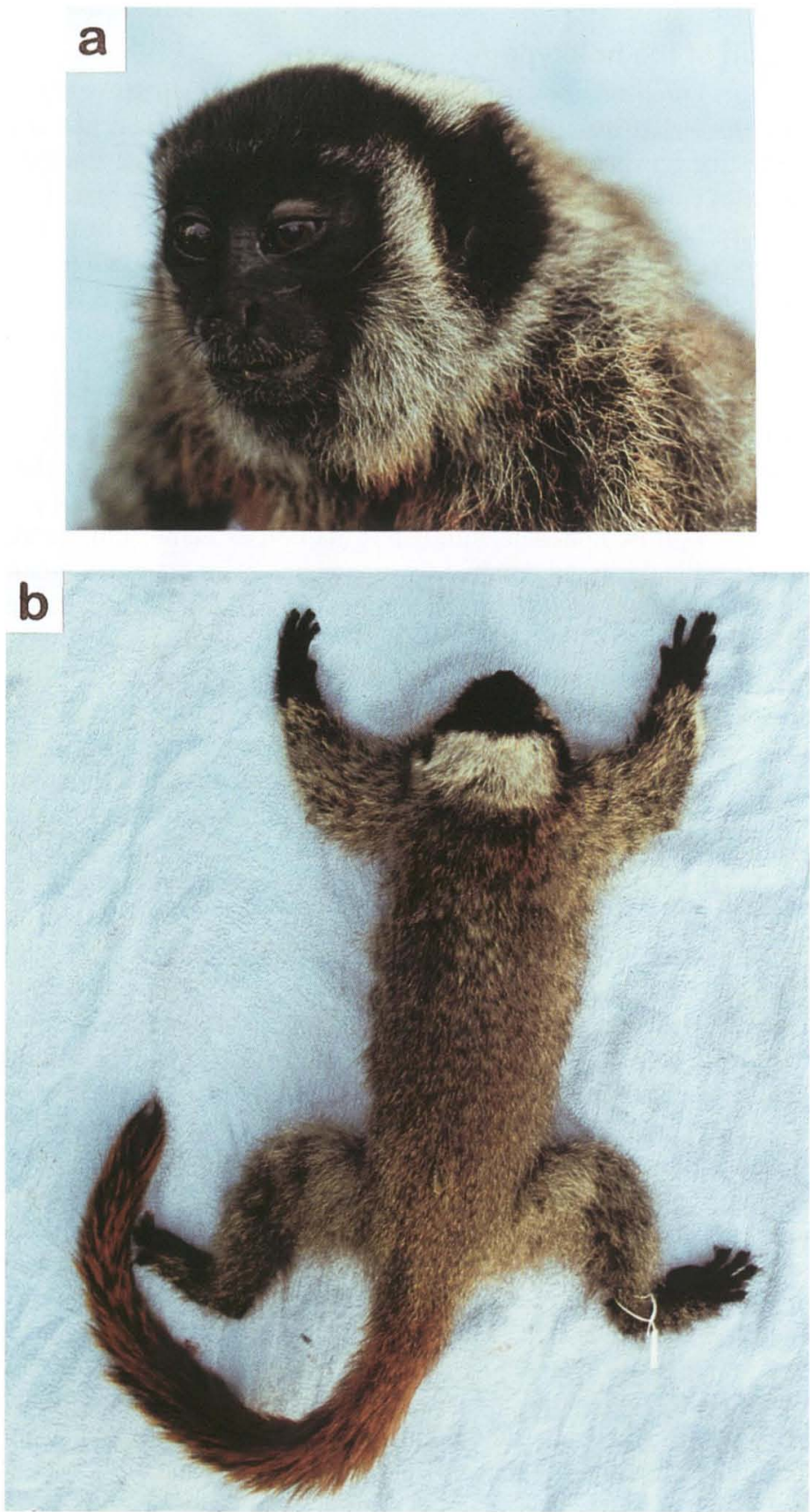

Fig. 4. (A) Head of fresh specimen of C. coimbrai (UFPB 1859); (B) dorsal view of fresh specimen of C. coimbrai (UFPB 1860).

Revta bras. Zool. 16 (2): 531 - 551, 1999 


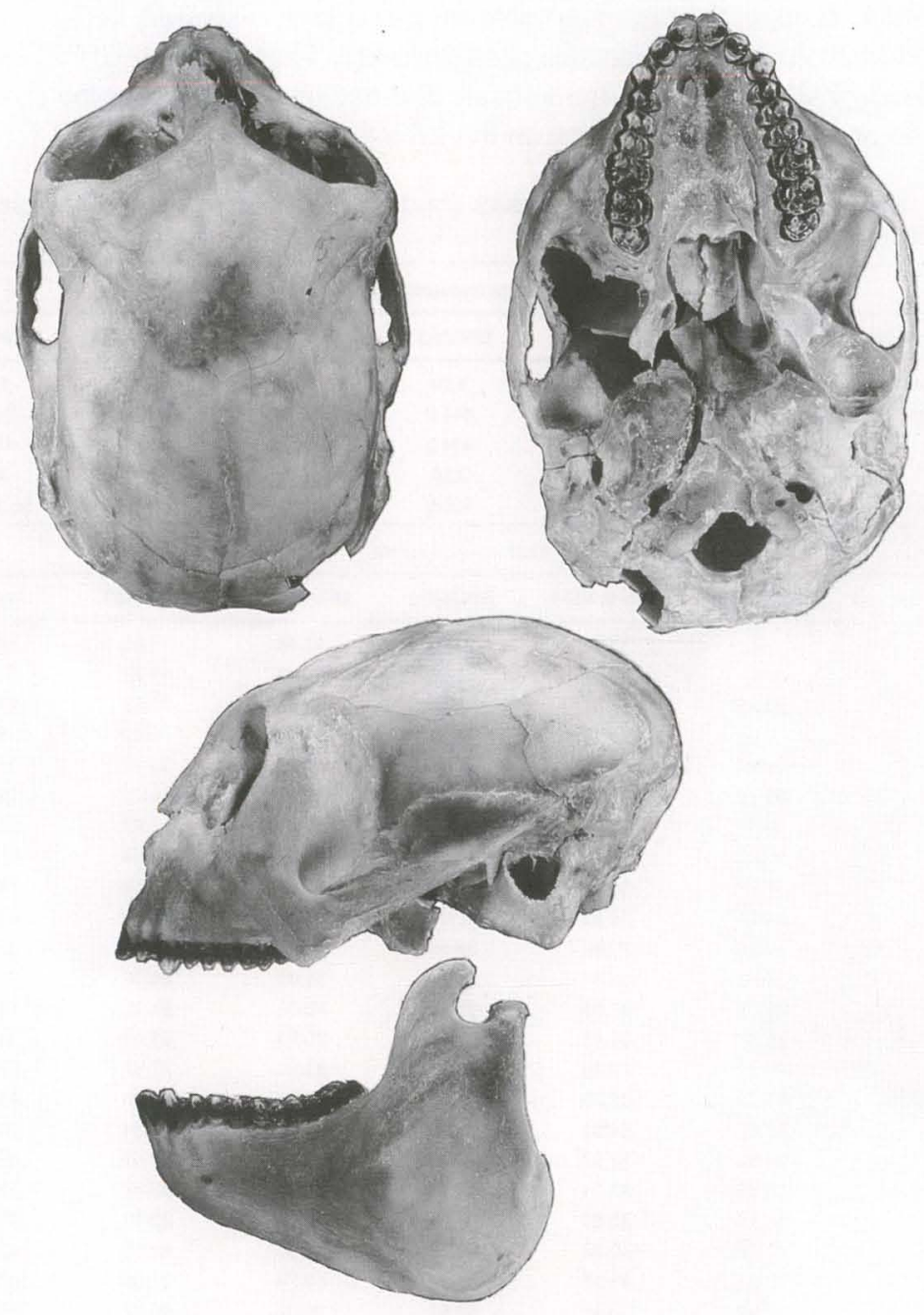

Fig. 5. Dorsal, ventral, and lateral views of skull and mandible of Callicebus coimbrai (holotype).

30550 , the zebra-like pattern is not clear on the anterior third of the back and the brownish colour on the posterior part of the back extends forwards occupying one-third of the trunk.

Skull. Variation in the measurements of the type specimens is shown in table I. In UFPB 1600 the pterygoid processes are finely shaped, but in MN 30550 the pterygoid processes are comparatively more developed and the parapterygoid 
processes more nearly triangular than rectangular, a fine projection is present on posteroventral edge; the mesopterygoid fossa is nearly U-shaped; in this specimen the infraorbital fissure is expanded anterolaterally. The specimen UFPB 1599 has two infraorbital foramina on the left side and one on the right but the remainder have two on the right side and one on the left side.

Table I. External and cranial measurements $(\mathrm{mm})$ of Callicebus coimbrai. See figure 1 for explanation of skull measurements.

\begin{tabular}{|c|c|c|c|c|c|c|}
\hline \multicolumn{7}{|c|}{ External measurements } \\
\hline Specimen No. & UFPB 1599 & UFPB 1600 & MN30550 & UFPB 1859 & UFPB 1860 & Average \\
\hline Body weight (Kg) & 1.21 & 1.03 & 1.20 & 1.15 & 1.30 & 1.20 \\
\hline Total length & 814.0 & 804.0 & 844.0 & 823.0 & 800.0 & 820.2 \\
\hline Tail & 467.0 & 461.0 & 484.0 & 471.0 & 453.0 & 468.7 \\
\hline Ear & 34.0 & 34.0 & 32.0 & 32.0 & 32.0 & 32.5 \\
\hline Hind foot s-c/u & $91.0-92.0$ & $98.0-99.0$ & 101.0 & $99.0-100.0$ & $95.0-97.0$ & $96.8-97.0$ \\
\hline \multicolumn{7}{|c|}{ Skull measurements } \\
\hline Measurement no. & UFPB 1599 & UFPB 1600 & MN30550 & UFPB 1859 & UFPB 1860 & Average \\
\hline 1 & - & 11.01 & 10.23 & 10.34 & 11.30 & 10.62 \\
\hline 2 & - & 12.12 & 11.62 & 11.48 & 11.98 & 11.69 \\
\hline 3 & 23.29 & 22.04 & 21.52 & 21.68 & 23.06 & 22.39 \\
\hline 4 & 6.35 & 5.48 & 6.82 & 5.46 & 6.04 & 6.17 \\
\hline 5 & 33.63 & 32.96 & 35.55 & 33.43 & 35.12 & 34.43 \\
\hline 6 & 29.38 & 27.52 & 30.64 & 30.38 & 29.27 & 29.92 \\
\hline 7 & 13.34 & 12.38 & 13.65 & 12.00 & 13.43 & 13.11 \\
\hline 8 & 15.69 & 14.96 & 14.84 & 16.89 & 16.44 & 15.97 \\
\hline 9 & 38.95 & 35.77 & 38.56 & 37.40 & 39.74 & 38.66 \\
\hline 10 & 43.01 & 39.62 & 40.21 & 40.43 & 43.62 & 41.82 \\
\hline 11 & 32.20 & 29.86 & 33.04 & 32.34 & 33.75 & 32.83 \\
\hline 12 & 34.62 & 32.91 & 35.66 & 35.40 & 36.26 & 35.49 \\
\hline 13 & 49.66 & 47.55 & 50.57 & 48.93 & 51.05 & 50.05 \\
\hline 14 & 30.07 & 29.48 & 32.11 & 30.85 & 31.43 & 31.12 \\
\hline 15 & 26.27 & 27.38 & 26.09 & 29.20 & 27.92 & 27.38 \\
\hline 16 & 65.20 & 63.26 & 68.25 & 64.78 & 65.45 & 65.92 \\
\hline 17 & 32.65 & 31.63 & 32.93 & 33.50 & 32.44 & 32.88 \\
\hline 18 & 34.62 & 32.07 & 33.22 & 31.28 & 34.00 & 33.28 \\
\hline 19 & 35.42 & 33.01 & 33.99 & 32.49 & 33.67 & 33.89 \\
\hline 20 & 38.11 & 35.80 & 37.58 & 37.39 & 39.73 & 38.20 \\
\hline 21 & 42.55 & 40.35 & 44.03 & 41.66 & 43.16 & 42.85 \\
\hline 22 & 12.61 & 11.97 & 11.24 & 11.74 & 11.96 & 11.89 \\
\hline 23 & 36.57 & 33.99 & 37.82 & 36.07 & 38.52 & 37.25 \\
\hline
\end{tabular}

Teeth. (Fig. 6) Specimens UFPB 1600, 1859 and MN 30550 have relatively unworn teeth except on the lingual side of the upper and lower premolars; UFPB 1860 is excessively worn, it being almost impossible to recognise the occlusal pattern of all teeth; the diastema between $\mathrm{I}^{1}$ and $\mathrm{I}^{2}$ in MN 30550 is conspicuous and larger than that between $\mathrm{I}^{2}$ and $\mathrm{C}$; the middle cusplet of the metaloph on $\mathrm{M}^{1}$ is obscure in UFPB 1859, is projected in MN 30550, and absent in UFPB 1600 (Fig. 6); the buccal cingulum on $\mathrm{M}^{1}$ is developed in UFPB 1600, 1859 and MN 30550 , and several stylar cusps are found on it; the lingual cingulum of $\mathrm{M}^{1}$ in UFPB 1600 extends distally and connects to the posthypocrista without connecting to the 

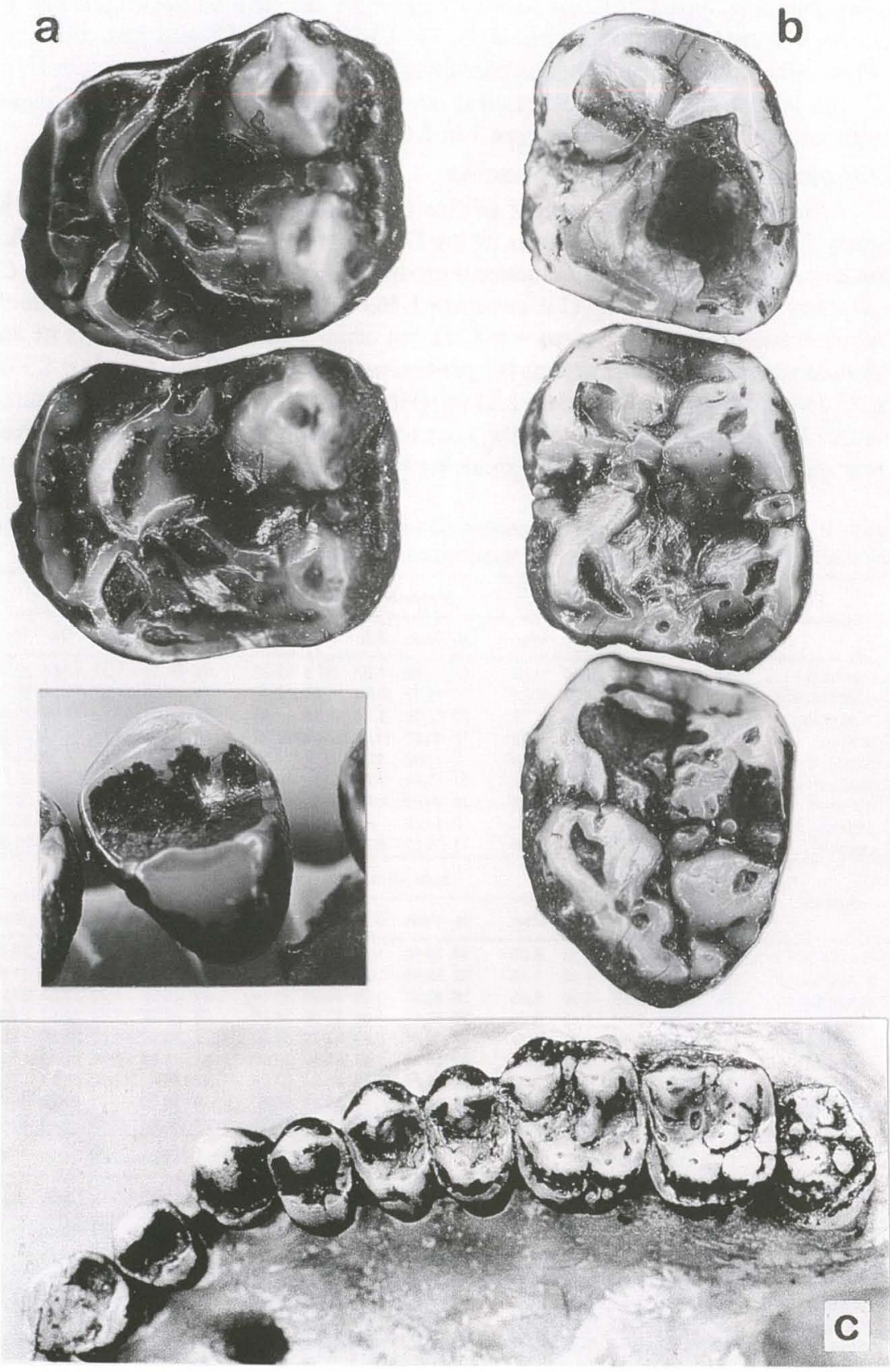

Fig. 6. Teeth of Callicebus coimbrai. (A) Left $\mathrm{M}^{1-2}$, and $\mathrm{I}^{2}$ (holotype); (B) Left $\mathrm{M}_{1-3}$ (holotype); (C) Left upper tooth row (UFPB 1600). 
hypocone (as in type 2 of KOBAYASH 1990); on $\mathrm{M}^{2}$ the dent on the middle part of metaloph is clearly seen in UFPB 1600, 1859 and MN 30550, and particularly in UFPB 1600 where the metaloph is transformed into two independent conules (Fig. $6 \mathrm{c})$; the buccal cingulum on $\mathrm{M}^{2}$ is less developed in MN 30550 and the lingual cingulum on this molar is of the type 1 of KOBAYASHI (1990).

\section{Comparison with other titi species}

Size. Average body weight of five C. coimbrai females, is $1.22 \mathrm{~kg}$. It is clearly larger than some members of the Donacophilus group (C. donacophilus donacophilus $=0.8 \mathrm{~kg}$ ), The Personatus group (C. cupreus cupreus $=1.106 \mathrm{~kg}, C$. c. discolor $=0.845-1.075 \mathrm{~kg}$, C. c. ornatus $=1.163-1.178)$, Moloch group $(C$. moloch female $=0.877 \mathrm{~kg}$, C. brunneus $=0.875)$, but smaller than other members of the Personatus and Torquatus groups $(C$. personatus male $=1.270 \mathrm{~kg}$, female $=1.378$ $\mathrm{kg}$, C. torquatus medemi female $=1.31 \mathrm{~kg})($ HershKovitz 1990). Cranial measurements (Tab. II) also indicate that the skull of C. coimbrai is slightly smaller than other members of the Personatus group but larger than that of other groups.

Table II. Basic statistical values for comparison of cranial measurements in nine different species of Callicebus. See figure 1 for explanation of skull measurements.

\begin{tabular}{|c|c|c|c|c|c|c|c|c|c|c|c|c|c|c|}
\hline \multirow{2}{*}{ Species } & \multicolumn{5}{|c|}{ Measurement no. 1} & \multicolumn{5}{|c|}{ Measurement no. 2} & \multicolumn{4}{|c|}{ Measurement no. 3} \\
\hline & $\mathrm{N}$ & Mean & S.D. & Min. & Max. & $N$ & Mean & S.D. & Min. & Max. & $\mathrm{N}$ & Mean & S.D. & Max. \\
\hline C. brunneus & 45 & 8.60 & 1.13 & 6.14 & 10.83 & 45 & 11.89 & 0.97 & 8.75 & 13.26 & 46 & 20.42 & $1.32 \quad 17.90$ & 22.88 \\
\hline C. h. hoffmannsi & 51 & 7.42 & 1.12 & 5.58 & 10.22 & 51 & 11.78 & 0.68 & 10.28 & 12.96 & 51 & 19.60 & 1.2716 .84 & 22.04 \\
\hline C. h. baptista & 28 & 7.38 & 1.09 & 4.73 & 10.52 & 28 & 12.00 & 1.13 & 6.71 & 14.66 & 28 & 19.04 & $1.06 \quad 17.09$ & 20.59 \\
\hline C. moloch & 74 & 7.40 & 1.01 & 5.70 & 8.52 & 75 & 12.50 & 0.87 & 10.98 & 13.59 & 74 & 19.34 & 1.1416 .71 & 22.53 \\
\hline C.cinerascens & 7 & 9.49 & 1.19 & 6.27 & 11.66 & 7 & 11.82 & 1.07 & 10.08 & 14.07 & 7 & 19.65 & 1.2916 .92 & 20.77 \\
\hline C.personatus & 41 & 10.43 & 1.45 & 7.41 & 13.25 & 41 & 13.20 & 1.07 & 10.59 & 15.34 & 42 & 23.17 & $1.92 \quad 18.73$ & 26.73 \\
\hline C. nigrifrons & 28 & 9.63 & 0.95 & 8.04 & 11.63 & 28 & 12.63 & 0.98 & 10.12 & 14.81 & 28 & 21.89 & 1.1518 .64 & 24.77 \\
\hline C. melanochir & 2 & 7.60 & - & 6.68 & 8.52 & 2 & 14.70 & - & 14.68 & 14.71 & 2 & 21.88 & -21.16 & 22.50 \\
\hline C. coimbrai & 3 & 10.62 & 0.59 & 10.23 & 11.30 & 3 & 11.69 & 0.26 & 11.48 & 11.98 & 4 & 23.39 & $0.92 \quad 21.52$ & 23.29 \\
\hline \multirow{2}{*}{ Species } & \multicolumn{5}{|c|}{ Measurement no. 4} & \multicolumn{5}{|c|}{ Measurement no. 5} & \multicolumn{4}{|c|}{ Measurement no. 6} \\
\hline & $\mathrm{N}$ & Mean & S.D. & Min. & Max. & $\mathrm{N}$ & Mean & S.D. & Min. & Max. & $\mathrm{N}$ & Mean & Min. & Max. \\
\hline C. brunneus & 46 & 5.20 & 0.62 & 3.58 & 6.90 & 47 & 30.50 & 1.19 & 28.52 & 33.29 & 47 & 26.10 & $1.43 \quad 23.23$ & 28.41 \\
\hline C. h. hoffmannsi & 51 & 5.50 & 0.71 & 3.80 & 7.16 & 52 & 32.99 & 1.27 & 30.31 & 36.12 & 52 & 26.52 & 1.5923 .38 & 31.47 \\
\hline C. h. baptista & 28 & 5.91 & 0.65 & 4.06 & 6.95 & 28 & 32.93 & 1.26 & 30.66 & 35.14 & 28 & 25.66 & $1.20 \quad 23.07$ & 27.94 \\
\hline C. moloch & 76 & 5.44 & 0.58 & 4.45 & 6.79 & 76 & 32.1 & 1.65 & 27.96 & 38.16 & 76 & 26.96 & 1.8923 .51 & 32.49 \\
\hline C.cinerascens & 7 & 5.26 & 0.33 & 4.74 & 5.77 & 7 & 32.57 & 0.89 & 31.73 & 34.26 & 7 & 28.16 & 1.9725 .56 & 31.04 \\
\hline C.personatus & 43 & 5.51 & 0.62 & 3.99 & 6.69 & 43 & 35.88 & 1.89 & 32.45 & 40.0 & 43 & 31. & $1.98 \quad 27.14$ & 37.85 \\
\hline nigrifrons & 28 & 5.49 & 0.64 & 4.11 & 7.04 & 28 & 34.96 & 1.53 & 31.84 & & 28 & 30 & $1.52 \quad 27.50$ & 33.94 \\
\hline C. melanochir & 2 & 6.48 & - & 6.46 & 6.4 & 2 & 35.49 & - & 35.48 & & 2 & & -32.55 & 33.45 \\
\hline C. coimbrai & 4 & 6.17 & 0.57 & 5.46 & 6.82 & 4 & 34.43 & 1.06 & 33.43 & 35.55 & 4 & 29.92 & $0.69 \quad 29.38$ & 30.64 \\
\hline \multirow{2}{*}{ Species } & \multicolumn{5}{|c|}{ Measurement no. 7} & \multicolumn{5}{|c|}{ Measurement no. 8} & \multicolumn{4}{|c|}{ Measurement no. 9} \\
\hline & $\mathrm{N}$ & Mean & S.D. & Min. & Max. & $N$ & Mean & S.D. & Min. & Max. & $\mathrm{N}$ & Mean & S.D. & Max. \\
\hline & 47 & 12.74 & 0.77 & 10.75 & & 47 & & 1.07 & 10.75 & & 47 & & 1.1932 .90 & 37.43 \\
\hline C. h. hoffmannsi & 52 & 13.31 & 1.01 & 11.17 & 15.15 & 52 & 12.59 & 1.00 & 10.71 & 15.90 & 52 & 36.98 & 1.3534 .41 & 39.98 \\
\hline C. h. baptista & 28 & 13.85 & 0.83 & 12.59 & 15.17 & 28 & 12.95 & 0.91 & 11.51 & 15.15 & 28 & 37.09 & 0.9535 .40 & 38.90 \\
\hline C. moloch & 76 & 12.47 & 1.07 & 9.07 & 15.04 & 76 & 13.45 & 0.86 & 10.84 & 15.20 & 76 & 36.24 & 1.4329 .81 & 39.65 \\
\hline C.cinerascens & 7 & 12.45 & 0.61 & 11.54 & 13.37 & 7 & 13.88 & 1.31 & 12.77 & 15.92 & 7 & 36.58 & 1.0134 .80 & 37.44 \\
\hline C.personatus & 43 & 12.63 & 0.94 & 10.80 & 15.00 & 43 & 15.47 & 1.23 & 12.82 & 17.81 & 43 & 40.07 & $1.56 \quad 36.79$ & 43.60 \\
\hline C. nigrifrons & 28 & 11.33 & 1.12 & 9.64 & 14.40 & 28 & 14.05 & 1.04 & 12.47 & 15.94 & 28 & 38.19 & 1.3035 .83 & 40.24 \\
\hline C. melanochir & 2 & 11.46 & - & 10.79 & 12.12 & 2 & 15.99 & - & 14.79 & 17.18 & 2 & 39.78 & - $\quad 39.45$ & 40.11 \\
\hline C. coimbrai & 4 & 13.11 & 0.75 & 12.00 & 13.65 & 4 & 15.97 & 0.90 & 14.84 & 16.89 & 4 & 38.66 & $0.97 \quad 37.40$ & 39.74 \\
\hline
\end{tabular}

Cont. 
Table II. Continued.

\begin{tabular}{|c|c|c|c|c|c|c|c|c|c|c|c|c|c|c|c|}
\hline \multirow{2}{*}{ Species } & \multicolumn{5}{|c|}{ Measurement no. 10} & \multicolumn{5}{|c|}{ Measurement no. 11} & \multicolumn{5}{|c|}{ Measurement no. 12} \\
\hline & $\mathrm{N}$ & Mean & S.D. & Min & Max. & $\mathrm{N}$ & Mean & S.D. & Min & Max. & $\mathrm{N}$ & Mean & S.D. & Min. & Max. \\
\hline C. brunneus & 46 & 39.65 & 1.54 & 35.55 & 42.71 & 47 & 30.28 & 0.91 & 27.14 & 32.20 & 47 & 34.36 & 0.87 & 32.88 & 36.44 \\
\hline C. h. hoffmannsi & 52 & 40.06 & 1.66 & 36.79 & 45.47 & 52 & 30.78 & 1.05 & 28.62 & 32.81 & 52 & 34.29 & 1.07 & 32.15 & 36.85 \\
\hline C. h. baptista & 28 & 39.30 & 1.20 & 36.89 & 41.05 & 28 & 30.71 & 1.03 & 28.62 & 32.76 & 26 & 34.18 & 0.89 & 32.79 & 35.93 \\
\hline C. moloch & 76 & 40.07 & 1.50 & 36.55 & 43.28 & 76 & 30.80 & 1.11 & 27.77 & 33.23 & 76 & 34.53 & 0.94 & 32.29 & 36.42 \\
\hline C.cinerascens & 7 & 40.66 & 1.67 & 37.79 & 43.34 & 7 & 30.87 & 0.90 & 29.50 & 31.87 & 7 & 35.17 & 1.34 & 33.68 & 37.63 \\
\hline C.personatus & 43 & 43.71 & 1.96 & 39.57 & 48.33 & 43 & 32.02 & 1.22 & 28.82 & 34.12 & 43 & 36.31 & 1.13 & 33.73 & 38.74 \\
\hline C. nigrifrons & 28 & 42.62 & 1.63 & 38.42 & 45.50 & 28 & 31.62 & 1.52 & 29.18 & 35.00 & 28 & 36.01 & 1.12 & 34.56 & 38.25 \\
\hline C. melanochir & 2 & 42.14 & - & 40.68 & 43.59 & 2 & 32.43 & - & 31.87 & 32.98 & 2 & 36.99 & - & 35.74 & 38.24 \\
\hline C. coimbrai & 4 & 41.82 & 1.75 & 40.21 & 43.62 & 4 & 32.83 & 0.71 & 32.20 & 33.75 & 4 & 35.49 & 0.68 & 34.62 & 36.26 \\
\hline \multirow{2}{*}{ Species } & \multicolumn{5}{|c|}{ Measurement no. 13} & \multicolumn{5}{|c|}{ Measurement no. 14} & \multicolumn{5}{|c|}{ Measurement no. 15} \\
\hline & $\mathrm{N}$ & Mean & S.D. & Min. & Max. & $\mathrm{N}$ & Mean & S.D. & Min. & Max. & $N$ & Mean & S.D. & Min. & Max. \\
\hline C. brunneus & 47 & 47.47 & 1.65 & 43.83 & 51.31 & 46 & 30.54 & 1.81 & 26.57 & 35.63 & 47 & 24.93 & 2.37 & 20.05 & 30.15 \\
\hline C. h. hoffmannsi & 52 & 48.00 & 1.80 & 43.16 & 51.23 & 51 & 31.73 & 2.07 & 25.41 & 35.66 & 52 & 24.66 & 1.71 & 20.82 & 30.38 \\
\hline C. h. baptista & 28 & 46.90 & 1.49 & 41.66 & 49.53 & 28 & 30.53 & 1.71 & 26.74 & 33.58 & 28 & 25.80 & 1.05 & 23.70 & 28.20 \\
\hline C. moloch & 74 & 47.26 & 1.92 & 42.77 & 53.67 & 73 & 30.99 & 1.82 & 26.66 & 35.85 & 73 & 26.62 & 1.70 & 21.00 & 29.61 \\
\hline C. cinerascens & 7 & 47.87 & 1.92 & 44.57 & 50.81 & 7 & 31.06 & 1.46 & 29.29 & 33.20 & 7 & 25.90 & 1.18 & 24.46 & 27.60 \\
\hline C.personatus & 43 & 52.61 & 1.68 & 49.45 & 56.36 & 42 & 33.42 & 1.75 & 29.96 & 36.87 & 43 & 27.26 & 1.60 & 24.71 & 31.56 \\
\hline C. nigrifrons & 28 & 51.28 & 1.81 & 48.14 & 55.24 & 28 & 33.54 & 2.08 & 29.20 & 37.67 & 25 & 27.60 & 1.39 & 25.20 & 30.88 \\
\hline C. melanochir & 2 & 50.87 & - & 50.41 & 51.32 & 2 & 32.07 & - & 31.23 & 32.90 & 2 & 27.28 & -2 & 25.28 & 29.27 \\
\hline C. coimbrai & 4 & 5.05 & 0.94 & 48.93 & 51.05 & 4 & 31.12 & 0.87 & 30.07 & 32.11 & 4 & 27.38 & 1.49 & 26.09 & 29.25 \\
\hline \multirow{2}{*}{ Species } & \multicolumn{5}{|c|}{ Measurement no. 16} & \multicolumn{5}{|c|}{ Measurement no. 17} & \multicolumn{5}{|c|}{ Measurement no. 18} \\
\hline & $N$ & Mean & S.D. & Min. & Max. & $\mathrm{N}$ & Mean & S.D. & Min. & Max. & $\mathrm{N}$ & Mean & S.D. & Min. & Max. \\
\hline C. brunneus & 47 & 60.91 & 2.06 & 57.34 & 65.85 & 46 & 30.97 & 1.31 & 28.09 & 33.84 & 46 & 31.36 & 2.11 & 28.51 & 42.85 \\
\hline C. h. hoffmannsi & 52 & 61.50 & 1.82 & 57.23 & 65.87 & 51 & 30.58 & 1.17 & 28.14 & 32.67 & 52 & 30.51 & 1.47 & 27.52 & 35.58 \\
\hline C. h. baptista & 28 & 60.75 & 1.36 & 58.13 & 63.82 & 28 & 30.92 & 0.92 & 29.48 & 32.69 & 28 & 30.34 & 1.10 & 27.41 & 32.19 \\
\hline C. moloch & 75 & 61.67 & 1.65 & 57.24 & 66.14 & 72 & 31.49 & 1.15 & 28.99 & 33.82 & 76 & 31.30 & 1.50 & 27.92 & 35.78 \\
\hline C. cinerascens & & 62.68 & 2.01 & 59.30 & 65.65 & 7 & 31.43 & 0.96 & 29.88 & 32.64 & 7 & 31.12 & 1.15 & 30.71 & 34.42 \\
\hline C.personatus & 43 & 68.42 & 1.96 & 64.00 & 72.26 & 42 & 34.24 & 1.30 & 31.23 & 37.55 & 43 & 35.36 & 1.62 & 32.31 & 38.85 \\
\hline C. nigrifrons & 28 & 66.06 & 1.82 & 62.46 & 69.80 & 28 & 34.37 & 1.52 & 31.25 & 36.85 & 28 & 33.28 & 1.38 & 30.12 & 36.27 \\
\hline C. melanochir & & 67.95 & - & 67.81 & 68.08 & 2 & 33.64 & - & 31.57 & 35.71 & 2 & 33.56 & - & 30.48 & 36.64 \\
\hline C. coimbrai & 4 & 65.92 & 1.58 & 64.78 & 68.25 & 4 & 32.88 & 0.46 & 32.44 & 33.50 & 4 & 33.28 & 1.45 & 31.28 & 34.62 \\
\hline \multirow{2}{*}{ Species } & \multicolumn{5}{|c|}{ Measurement no. 19} & & Measure & ement $\mathrm{r}$ & no. 20 & & & Measure & ment $n$ & no. 21 & \\
\hline & $N$ & Mean & S.D. & Min. & Max. & $\mathrm{N}$ & Mean & S.D. & Min. & Max. & $N$ & Mean & S.D. & Min. & Max. \\
\hline C. brunneus & 45 & 33.81 & 1.54 & 29.34 & 37.71 & 45 & 35.98 & 1.53 & 31.56 & 38.71 & 46 & 39.71 & 1.52 & 36.29 & 43.89 \\
\hline C. h. hoffmannsi & 51 & 34.63 & 1.30 & 31.65 & 38.23 & 51 & 36.38 & 1.43 & 34.01 & 39.91 & 51 & 40.61 & 1.64 & 37.50 & 44.28 \\
\hline C. h. baptista & 28 & 33.54 & 1.42 & 31.18 & 37.03 & 27 & 35.40 & 1.38 & 33.21 & 38.49 & 28 & 39.85 & 1.31 & 37.43 & 43.36 \\
\hline C. moloch & 72 & 34.05 & 1.46 & 30.11 & 37.67 & 74 & 37.02 & 1.59 & 32.18 & 42.45 & 76 & 40.15 & 1.54 & 35.65 & 43.71 \\
\hline C.cinerascens & 7 & 35.60 & 1.50 & 33.50 & 37.22 & 7 & 37.39 & 1.35 & 35.12 & 39.15 & 7 & 40.85 & 1.46 & 37.87 & 42.49 \\
\hline C.personatus & 43 & 36.36 & 1.74 & 31.98 & 39.96 & 43 & 39.63 & 1.97 & 35.79 & 44.27 & 43 & 45.64 & 1.96 & 41.88 & 50.25 \\
\hline C. nignifrons & 28 & 35.57 & 1.22 & 33.33 & 37.54 & 28 & 38.26 & 2.10 & 33.37 & 43.91 & 28 & 44.41 & 1.78 & 40.75 & 47.58 \\
\hline C. melanochir & & 35.32 & - & 33.72 & 36.91 & & 39.35 & - & 38.09 & 40.61 & & 45.22 & - & 45.10 & 45.34 \\
\hline C. coimbrai & & 33.89 & 1.21 & 32.49 & 35.42 & & 38.20 & 1.06 & 37.39 & 39.73 & 4 & 42.85 & 1.00 & 41.66 & 44.03 \\
\hline & & Measure & ement & no. 22 & & & Measu & ment & no. 23 & & & & & & \\
\hline & $\mathrm{N}$ & Mean & S.D. & Min. & Max. & $N$ & Mean & S.D. & Min. & Max. & & & & & \\
\hline C. brunneus & 46 & 11.28 & 0.70 & 9.13 & 13.15 & 46 & 34.31 & 2.32 & 29.11 & 39.44 & & & & & \\
\hline C. h. hoffmannsi & 51 & 11.87 & 0.80 & 9.55 & 13.36 & 52 & 35.29 & 2.18 & 29.79 & 40.33 & & & & & \\
\hline C. h. baptista & 28 & 11.59 & 0.65 & 10.65 & 13.39 & 27 & 33.24 & 1.91 & 30.22 & 37.27 & & & & & \\
\hline C. moloch & 76 & 11.37 & 0.78 & 9.23 & 13.46 & 76 & 35.22 & 1.98 & 29.16 & 39.46 & & & & & \\
\hline C.cinerascens & 7 & 11.88 & 0.63 & 10.77 & 12.64 & 7 & 35.40 & 2.77 & 32.20 & 40.27 & & & & & \\
\hline C.personatus & 43 & 13.22 & 0.96 & 11.89 & 15.38 & 43 & 38.24 & 2.44 & 33.89 & 44.02 & & & & & \\
\hline C. nigrifrons & 28 & 11.91 & 0.98 & 10.22 & 13.66 & 28 & 36.07 & 2.31 & 31.23 & 40.96 & & & & & \\
\hline C. melanochir & 2 & 14.20 & - & 12.95 & 15.44 & 2 & 40.78 & - & 40.32 & 41.24 & & & & & \\
\hline C. coimbrai & 4 & 11.89 & 0.57 & 11.24 & 12.61 & 4 & 37.25 & 1.12 & 36.07 & 38.52 & & & & & \\
\hline
\end{tabular}


Pelage. In general appearance C. coimbrai is easily identified as a member of the Personatus group by both the blackish head and the orange or brownish tail (Fig. 3). Within the Personatus group, the new form is sharply distinguished from Barbara Brown's titi C. barbarabrownae and SPIX's (1823) figure of C. gigot (HERSHKOVITZ 1990), by its black head and crown with a clear line demarcating the whitish back of the crown and nape, as well as the buffy preauricular stripe and brownish tail. C. coimbrai differs from the other three members of the Personatus group by the buffy colour of its pelage, the zebra-striped pattern of the anterior half of the back, the well-defined whitish band across the back of the crown and nape, and the buffy preauricular stripe.

Teeth. According to KOBAYASHI (1990), each group of titis show different tendencies in the occlusal pattern of the upper molars. In the Torquatus group the occlusal pattern is relatively smooth and simple. There are numerous cusps and conules and the pattern is more complicated in the Donacophilus, Cupreus and Moloch groups. The number of small cusps and conules is largest, and the pattern most uneven and variable in the Personatus group. In $C$. coimbrai this trend is actually emphasised. For example, the metaloph on $\mathrm{M}^{1}$ and $\mathrm{M}^{2}$ is fragmented, does not form a continuous ridge, and looks like two conical conules. This structural complexity is found in the other teeth as well.

\section{Craniometric comparison by multivariate analyses}

Distance analysis by Q-mode correlation coefficients and principal component analysis showed the new form to be clearly separated from the other members of the Personatus and Moloch groups analysed. In the distance analysis by Q-mode correlation coefficients, both clustering and ordination are applied. Table III shows the similarity matrix from Q-mode correlation coefficients. The dendrogram using the furthest neighbours method presents a hierarchical scheme for nine forms of the Personatus and Moloch groups, including coimbrai (Fig. 7). These forms can be divided into major clusters. The first includes $C$. h. hoffmannsi, $C$. h. baptista, $C$. moloch and $C$. cinerascens. The second includes $C$. personatus, $C$. nigrifrons, $C$. melanochir, C. brunneus and C. coimbrai. The morphometric distance between the new form and the other members of the second major cluster was larger than that between every pair within the first major cluster except $C$. brunneus.

Table III. Similarity matrix from Q-mode correlation coefficients obtained from skull measurements of species of Callicebus (Personatus and Moloch groups).

\begin{tabular}{|c|c|c|c|c|c|c|c|c|c|}
\hline & $\begin{array}{c}\text { C. } \\
\text { brunneus }\end{array}$ & $\begin{array}{c}\text { C. } h \text {. } \\
\text { hoffmannsi }\end{array}$ & $\begin{array}{c}\text { C. h. } \\
\text { baptista }\end{array}$ & $\begin{array}{c}c . \\
\text { moloch }\end{array}$ & $\begin{array}{c}\text { C. } \\
\text { cinerascens }\end{array}$ & $\begin{array}{c}\text { C. } \\
\text { personatus }\end{array}$ & $\underset{\text { nigrifrons }}{C .}$ & $\underset{\text { melanochir }}{\mathrm{C}}$ & $\underset{\text { coimbrai }}{\text { C. }}$ \\
\hline C. brunneus & 1.0000 & 0.2533 & 0.1503 & 0.0510 & -0.0101 & -0.1408 & -0.3472 & -0.5579 & 0.2025 \\
\hline C. h. hoffmannsi & 0.2533 & 1.0000 & 0.5589 & -0.0392 & 0.0137 & -0.2291 & -0.4687 & -0.4867 & -0.2123 \\
\hline C. h. baptista & 0.1503 & 0.5589 & 1.0000 & 0.3429 & -0.1972 & -0.7637 & -.6953 & -0.3210 & 0.1627 \\
\hline C. moloch & 0.0510 & -0.0392 & 0.3429 & 1.0000 & 0.2965 & -0.4565 & -0.2696 & -0.1525 & -0.0995 \\
\hline C. cinerascens & -0.0101 & 0.0137 & -0.1972 & 0.2965 & 1.0000 & 0.1660 & -0.0911 & 0.0822 & -0.6614 \\
\hline C. personatus & -0.1408 & -0.2291 & -0.7637 & -0.4565 & 0.1660 & 1.0000 & 0.6947 & -0.0935 & -0.2508 \\
\hline C. nignifrons & -0.3472 & -0.4687 & -0.6953 & -0.2696 & -0.0911 & 0.6947 & 1.0000 & 0.0528 & -0.1902 \\
\hline C. melanochir & -0.5579 & -0.4867 & -0.3210 & -0.1525 & 0.0822 & -0.0935 & 0.0528 & 1.0000 & -0.2178 \\
\hline C. coimbrai & 0.2025 & -0.2123 & 0.1627 & -0.0995 & -0.6614 & -0.2508 & -0.1902 & -0.2178 & 1.0000 \\
\hline
\end{tabular}




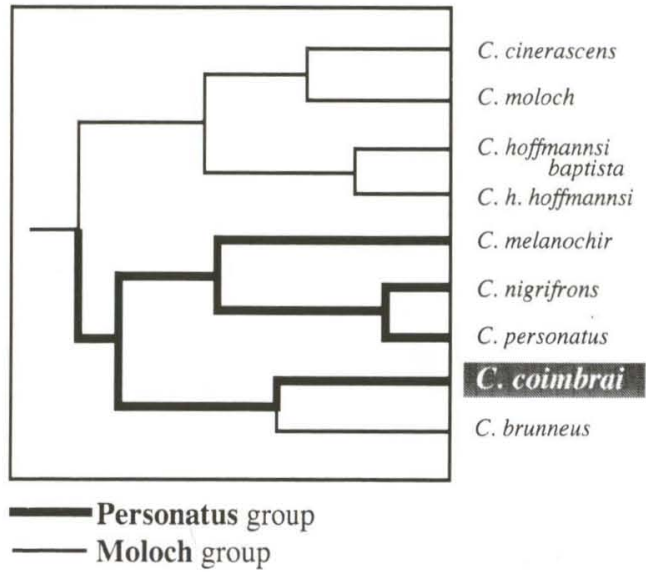

Fig. 7. Dendrogram by the furthest neighbours method of nine species of the Personatus and Moloch groups.

Table IV lists the Eigenvectors for the first three axes which resulted from the quantification theory model 4 of the Q-mode correlation coefficients, represented in three dimensions in figure 8. In this scattergram, the scatter pattern of each form shows some slight differences compared to the results of the clustering. $C$. brunneus is closer to the members of the first major cluster than the new form. $C$. coimbrai is clearly separated from any other form by the third axis.

Table IV. Eigenvectors and eigenvalues obtained from skull measurements of Callicebus species of the personatus and moloch groups resulting from quantification theory model 4 by Q-mode correlation coefficients.

\begin{tabular}{lcccccc}
\hline \multicolumn{1}{c}{ Species } & 1 & 2 & 3 & 4 & 5 & 6 \\
\hline C. brunneus & -0.22451 & -0.21940 & -0.03544 & -0.19917 & 0.26916 & 0.73263 \\
C. h. hoffmannsi & -0.30124 & -0.09582 & -0.28402 & -0.28969 & -0.51872 & 0.08332 \\
C. h. baptista & -0.46355 & 0.11351 & -0.03232 & 0.17039 & -0.36930 & -0.32433 \\
C. moloch & -0.17856 & 0.12997 & -0.15629 & 0.47965 & 0.38939 & -0.21565 \\
C. cinerascens & 0.08001 & 0.11332 & -0.44979 & 0.01966 & 0.42908 & -0.06259 \\
C. personatus & 0.44284 & -0.36445 & -0.11884 & -0.47710 & 0.06457 & -0.44364 \\
C. nigrifrons & 0.51209 & -0.29542 & 0.07769 & 0.58202 & -0.35616 & 0.23809 \\
C. melanochir & 0.32963 & 0.80353 & 0.20699 & -0.21262 & -0.11388 & 0.14947 \\
C. coimbrai & -0.19672 & -0.18525 & 0.79202 & -0.07314 & 0.20586 & -0.15765 \\
\hline Eigenvalues & 6.31905 & 3.83912 & 3.36905 & 1.34611 & 1.15754 & 0.32408 \\
\hline
\end{tabular}

In the PCA Eigenvectors, Eigenvalues, proportions and cumulative proportions for the first five principal components are listed in table $\mathrm{V}$. The first five factors have Eigenvalues greater than 1.0, and their cumulative proportion of the total variance was $76.1 \%$. It is quite probable that these principal components were responsible for a large part of the total variation. As is easily seen, the first principal component represents the overall cranial size (size factor), since all elements of the Eigenvector are greater than zero and display little differences in their values. In spite of difficulty in their translation, the other four principal components can be 


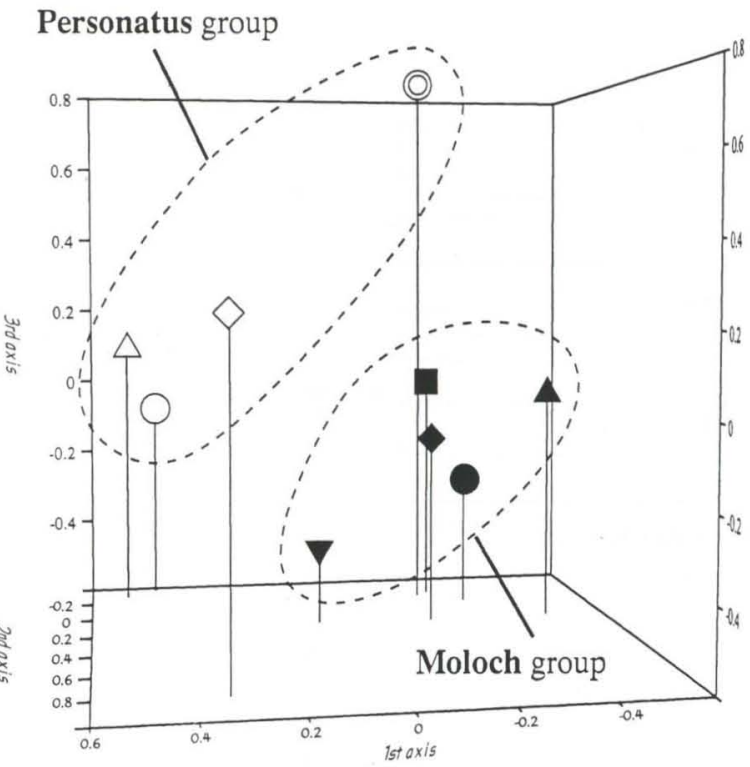

\begin{tabular}{|l|}
\hline C. brunneus \\
C.h.hoffmannsi \\
C.h.baptista \\
$\nabla$ c.moloch \\
c. cinerascens \\
$\triangle$ c.personatus \\
c.nigrifrons \\
C.melanochir \\
(O) c. coimbrai
\end{tabular}

Fig. 8. Three-dimensional representation of the Eigenvectors from table IV, calculated from skull measurements of specimens of Callicebus of the Personatus and Moloch groups.

Table V. Eigenvectors, eigenvalues, proportions and cumulative proportions for the first to five principal components resulting from PCA of skull measurements of Callicebus species of the personatus and moloch groups.

\begin{tabular}{cccccc}
\hline Measurement & PC1 & PC2 & PC3 & PC4 & PC5 \\
\hline 1 & 0.15684 & 0.25296 & -0.11081 & 0.59649 & 0.01048 \\
2 & 0.18579 & 0.11603 & -0.22858 & -0.21586 & 0.11865 \\
3 & 0.21908 & 0.25990 & -0.22811 & 0.34048 & 0.08613 \\
4 & 0.06691 & -0.37813 & -0.22286 & -0.18492 & 0.18748 \\
5 & 0.22675 & -0.19137 & 0.03336 & 0.07170 & -0.05055 \\
6 & 0.24306 & 0.10685 & 0.00737 & -0.03040 & -0.18053 \\
7 & -0.02999 & -0.46066 & -0.08269 & 0.28773 & 0.58773 \\
8 & 0.17319 & 0.27769 & -0.19926 & -0.18372 & 0.02827 \\
9 & 0.24949 & -0.20571 & -0.07444 & 0.09488 & 0.05497 \\
10 & 0.26033 & -0.09185 & -0.06418 & -0.09719 & 0.01211 \\
11 & 0.16832 & -0.15458 & 0.47080 & 0.21882 & 0.10307 \\
12 & 0.20507 & -0.03510 & 0.45626 & 0.04055 & -0.05207 \\
13 & 0.26220 & -0.07164 & 0.03334 & 0.11858 & -0.20395 \\
14 & 0.18535 & -0.33334 & 0.17297 & -0.08659 & -0.41075 \\
15 & 0.12276 & 0.29166 & 0.31334 & -0.28864 & 0.55386 \\
16 & 0.27055 & 0.06701 & 0.05680 & 0.01986 & 0.03904 \\
17 & 0.21482 & 0.23039 & 0.28744 & -0.06467 & 0.13649 \\
18 & 0.23468 & 0.05445 & -0.09194 & -0.09174 & -0.04811 \\
19 & 0.22267 & -0.15360 & -0.03604 & -0.06417 & 0.06096 \\
20 & 0.23121 & -0.09114 & -0.00628 & -0.21312 & 0.00985 \\
21 & 0.26366 & 0.02107 & -0.06894 & -0.00199 & -0.01550 \\
22 & 0.19462 & -0.00357 & -0.21104 & 0.18501 & 0.01132 \\
23 & 0.20880 & -0.06767 & -0.26917 & -0.23510 & 0.04064 \\
\hline Eigenvalue & 12.07030 & 1.83222 & 1.42754 & 1.18028 & 1.00206 \\
Proportion & 0.52480 & 0.07966 & 0.06207 & 0.05132 & 0.04357 \\
pumulative & 0.52480 & 0.60446 & 0.66652 & 0.71784 & 0.76141 \\
\hline
\end{tabular}

Revta bras. Zool. 16 (2): 531 - 551, 1999 
interpreted as shape factors, since each principal component had both positive and negative elements of Eigenvectors. Table VI lists the mean scores for the second to fourth principal components. The three-dimensional scattergram in figure 9 was drawn using the means of the first three principal component scores. In this scattergram, the overall pattern of the individual forms corresponds approximately to the results of clustering based on the Q-mode correlation coefficients. However, the plot of $C$. coimbrai is comparatively well separated from those of the other forms.

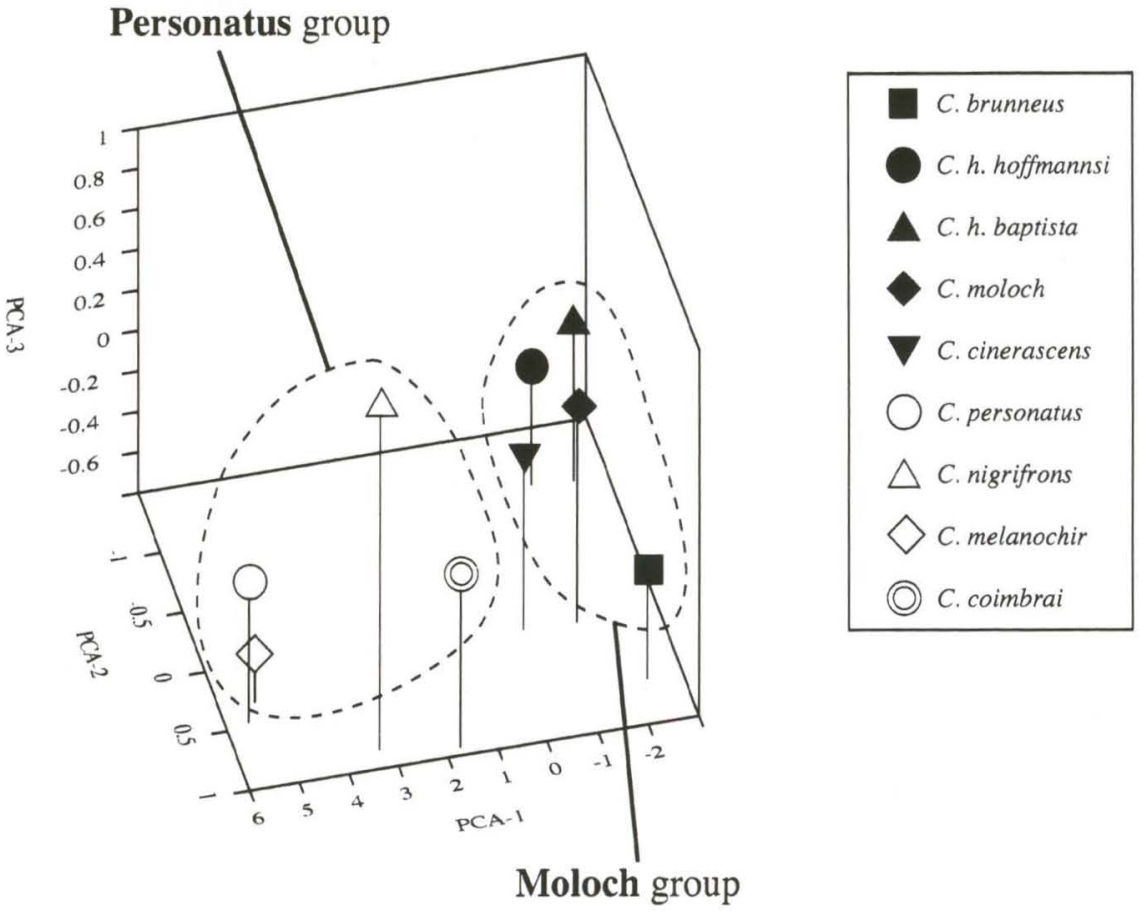

Fig. 9. Three-dimensional representation of the means of the first three principal component scores of skull measurements of specimens of Callicebus of the Personatus and Moloch groups.

Table VI. Mean scores for the principal components resulting from PCA of skull measurements of Callicebus species of the personatus and moloch groups.

\begin{tabular}{lccccc}
\hline \multicolumn{1}{c}{ Species } & First & Second & Third & Fourth & Fifth \\
\hline C. brunneus & -2.291 & 0.641 & -0.303 & 0.391 & -0.158 \\
C. h. hoffmannsi & -1.495 & -1.046 & -0.283 & 0.518 & -0.190 \\
C. h. baptista & -2.382 & -1.009 & -0.045 & 0.108 & 0.876 \\
C. moloch & -1.399 & 0.099 & 0.209 & -0.804 & 0.158 \\
C. cinerascens & -0.321 & 0.087 & 0.023 & -0.779 & -0.144 \\
C. personatus & 5.540 & 0.460 & -0.193 & 0.431 & -0.018 \\
C. nigrifrons & 3.247 & 0.851 & 0.849 & -0.183 & -0.684 \\
C. melanochir & 5.289 & 0.307 & -0.654 & -1.597 & 0.061 \\
C. coimbrai & 1.707 & 0.938 & -0.031 & 0.771 & 0.797 \\
\hline
\end{tabular}




\section{DISCUSSION}

If the taxonomic arrangement of HershKovitz $(1988,1990)$ is followed, $C$. coimbrai would be considered a subspecies of C. personatus. HERSHKOVITZ (1990), using mainly the colour variation of the pelage, considered all forms of the Personatus group to be subspecies. However, KOBAYASHI (1995) suggested that the craniometric differences found among the members of the Personatus group $(C$. barbarabrownae was not studied) argues for their separation as species rather than subspecies. Although the reproductive species concept is followed, it is in this case impossible to confirm the reproductive isolation of the new titi in the wild because of habitat destruction and fragmentation. It is here argued that morphological differences and the absence of intermediate forms are indirect evidence of reproductive isolation. Callicebus coimbrai is, therefore, described as a new species for the following reasons:

\section{Distribution}

The distribution of the new form is allopatric. The habitat may have been contiguous with that of the nearest forms, $C$. barbarabrownae to the west and $C$. melanochir to the south, before the widespread destruction and fragmentation of their forests through human activities (COIMBRA-FILHO \& CÂMARA 1996), but evidence concerning this will be difficult to obtain. Furthermore, there are striking differences in the habitat of the three forms. The Atlantic forest in the south of the state Bahia (C. melanochir) is different to that in Sergipe, and the semiarid habitat of C. barbarabrownae is very different from the Atlantic forest, although in the past more humid forests were widespread in the region (COIMBRA-FILHO \& CÂMARA 1996). The new form is clearly distinguishable, and there is no evidence of hybridisation or transitional phenotypes. The presence of melanochir-like phenotypes within the C. coimbrai populations would be an indication of gene flow and would argue for their classification as subspecies, but this is not the case. Recently, MARINHO-FILHO \& VERISSIMO (1997) reported new records of C. barbarabrownae at about the same latitude of $C$. coimbrai but further to the west, in small forest enclaves in the Caatinga of Canudos, Jeremoabo and Monte Santo, localities of Northern Bahia. Further information must be obtained from the region south of the Rio Real to the Rio Paraguaçu in Bahia, where scattered and isolated populations of titi monkeys undoubtedly still exist, but their identity has still to be ascertained (OLIVER \& SANTOS 1991). The forms in such as Callithrix and Leontopithecus, which show similar patterns in terms of their distribution and differentiation in the Atlantic forest, are classified as species (KINZEY 1982; MITTERMEIER et al. 1988; NATORI 1989; RYLANDS et al. 1993).

\section{Dental morphology}

The new form evidently shows a unique dental morphology as mentioned above. Differences in dental morphology are usually given considerable importance because the traits are conservative and subject to strong selection pressures.

\section{Skull shape}

As shown by the multivariate analyses, the craniometric shape of the new form is quite differentiated, and the degree of differentiation from the other members 
of the Personatus group corresponds to that found between species of the other Callicebus groups.

For similar reasons, it is also considered that the other members of the Personatus group are not subspecies but full species (paper in preparation).

\section{Material examined}

Callicebus personatus. Baixo Rio Suaçuí, Minas Gerais, MZUSP 5931-2; Colatina, Espírito Santo, MZUSP 2219-27; Estrada Linhares-São Mateus, Espírito Santo, MN 21051; Fazenda Afluente Branco do Sul, Espírito Santo, MN 21053; Fazenda Dez de Agosto, Espírito Santo, MN 21054; Fazenda Monte Verde, 24 km SE of Venda Nova, Espírito Santo, UFPB 412; Fazenda São José, Espírito Santo, MN 21052; Lagoa Japurá, Espírito Santo, MN 2478; Rio Doce, Espírito Santo, MZUSP 2409-3; Sooretama, Espírito Santo, MZUSP 11142, 11148, 11152, 11164 , 11711-4, 11803-5; São Domingos, Espírito Santo, Minas Gerais (3 specimens not numbered); Teófilo Otoni, Minas Gerais, MZUSP 2712-4, AMNH 139924, Locality unknown-MN 1428, MN 2479, USNM 240086.

Callicebus nigrifrons. Bairro dos Alves, Monte Alegre, São Paulo, MZUSP 6300-2; Fazenda Itaquerê, Boa Esperança do Sul, São Paulo, MZUSP 9812-3; Fazenda Tapera, Juiz de Fora, Minas Gerais, AMNH 133918; Franca, São Paulo, MZUSP 1077; Itatiaia, Rio de Janeiro, MN 25897, MZUSP 7426-30, 19548; Itatiba, MZUSP 3712; Ituéta, Rio Doce, Minas Gerais, MN 11978-9; Lins, Rio Tietê, São Paulo, MZUSP 5901, FMNH 52337; Macieiras, Serra do Itatiaya, São Paulo, AMNH 61539; Mato Grosso, Barretos, São Paulo, MZUSP 1419-21; Patos, Minas Gerais, AMNH 133685-6; Rio Doce (right bank), Minas Gerais, MZUSP 5928-30; São João da Glória, Passos, Minas Gerais, MN 21064-6, 25898; São Paulo, São Paulo,AMNH 36812-3; Uberlândia, Minas Gerais, AMNH 518219-20; UHE Nova Ponte, Nova Ponte, Minas Gerais, UFPB 1606, 2223, 2224, 2219; Visconde de Soutelo, São Paulo, MZUSP 6562; Locality unknown - MN 1357, 1866, 2477, 30174-8.

Callicebus melanochir. Fazenda Imbaçuaba, $30 \mathrm{~km} \mathrm{~N}$ de Prado, Bahia, UFPB 2217; Ilhéus, Bahia, MN 11201; Locality unknown - MN 11049, MZUSP 3884.

Callicebus barbarabrownae. Bandeira de Melo, Rio Paraguaçu, Bahia, FMNH 20444; Mirorós, Ibipeba, Bahia, UNB 1510.

ACKNOWLEDGMENTS. Useful data on distributions and ecology of titi monkeys were offered by the following: A.F. Coimbra-Filho (ex-director of the Centro de Primatologia do Rio de Janeiro -CPRJ/FEEMA, Brazil), E. Wilson (Sytech 3, Brazil), and Leila M. Pessoa and João A. de Oliveira (Universidade Federal do Rio de Janeiro- UFRJ, Brazil). Special thanks are due to T. Setoguchi, Kyoto University, for supporting this study. The authors are grateful to $\mathrm{M}$. Natori, Okayama Science University, and N. Shigehara and M. Aimi, Kyoto University, Japan, who critically reviewed earlier manuscripts of this paper. We also are most grateful to the following people and their staff for the many courtesies received during visits to their collections: Leda Dau (retired director of the Museu Nacional, Rio de Janeiro, Brazil), S.A. Marques and José de Sousa e Silva Junior (Museu Paraense Emílio Goeldi, Brazil), P.E. 
Vanzolini and R.L. Spieker (Museu de Zoologia da Universidade de São Paulo, Brazil), J. Marinho-Filho (Universidade de Brasilia, Brazil), R.W. Thorington, Jr. (National Museum of Natural History, U.S.A.), B.D. Patterson and the late P. Hershkovitz (Field Museum of Natural History, U.S.A.), and G. G. Musser (American Museum of Natural History, U.S.A.). Last not least, special thanks are due to Anthony B. Rylands who critically reviewed the final version of the manuscript. This study was financed by the Ministry of Education of Japan, the Ishida Foundation of Japan, the MacArthur Foundation of USA (to S.K.) and the Conselho Nacional de Desenvolvimento Científico e Tecnológico (CNPq), of Brazil (to A. Langguth).

\section{REFERENCES}

CoImBra-Filho, A.F. \& I. DE G. CÂMARA. 1996. Os Limites Originais do Bioma Mata Atlântica na Região Nordeste do Brasil. Rio de Janeiro, Fundação Brasileira para a Conservação da Natureza, 86p.

HershKovitz, P. 1988. Origin, speciation and distribution of South American titi monkeys, genus Callicebus (family Cebidae, Platyrrhini). Proc. Acad. Nat. Sci. Philadephia 140 (1): 240-272.

- 1990. Titis, New World monkeys of the genus Callicebus (Cebidae, Platyrrhini): A preliminary taxonomic review. Fieldiana, Zool. N.S., 55: 1-109. KINZEY, W.G. 1982. Distribution of primates and forest refuges, p.456-482. In: G.T. PRANCE (Ed.). Biological Diversification in the Tropics. New York, Columbia University Press, 714p.

KoBAYASHI, S. 1990. A morphological study of upper first and second molars in the genus Callicebus. Jour. Anthrop. Soc. Nippon 98 (2): 121-135.

. 1995. A phylogenetic study of titi monkeys, genus Callicebus, based on cranial measurements: I. Phyletic groups of Callicebus. Primates 36 (1): 101-120.

MARINHO-FILho, J. \& E.W. Veríssimo. 1997. The rediscovery of Callicebus personatus barbarabrownae in Northeastern Brazil with a new Western limit for its distribution. Primates 38 (4): 429-433.

MARTIN, R. \& K. SALLER. 1957. Lehrbuch der Anthropologie. Stuttgart, Gustav Fischer Verlag, Vol. 1.

MitTeRmeIER, R.A.; A.B. RYlands \& A.F. CoImBRA-FilHo. 1988. Systematics: species and subspecies - an update, p.13-75. In: R.A. MitTERMEIER; A.B. RYlands; A.F. COIMBRA-FilHo \& G.A.B. DA FonseCA (Eds). Ecology and Behavior of Neotropical Primates. Washington D.C., World Wildlife Fund, Vol. 2, 610p.

NATORI, M. \& T. HANIHARA. 1989. Morphological variations of cranium within the genus Leontopithecus. Primates 30 (2): 265-268.

Oliver, W.L.R. \& I.B. SANTOS. 1991. Threatened endemic mammals of the Atlantic forest region of south-east Brazil. Wildl. Preserv. Trust Spec. Scient. Report (4): 1-123.

REDFORD, K.H. \& J.F. EISENBERG. 1992. Mammals of the Neotropics 2: The Southern Cone. Chicago, University of Chicago Press, 430p.

RizzINI, C.T.; A.F. COIMBRA-FILHO \& A. HouAISS. 1988. Ecossistemas Brasileiros. Rio de Janeiro, Editora Index, 199p. 
RYlands, A.B.; A.F. CoImBra-Filho \& R.A. MitTermeIER. 1993. Systematics, distributions and some notes on the conservation status of the Callitrichidae, p.11-77. In: A.B. RYLANDS (Ed.) Marmosets and Tamarins: Systematics, Behaviour, and Ecology. Oxford, Oxford University Press, 396p.

SNEATH, P.H.A. \& R.R. SOKAL. 1973. Numerical Taxonomy: The Principles and

Practice of Numerical Classification. San Francisco, Freeman and Co., 573p. SPIX, J. DE. 1823. Simiarum et Vespertilionum Brasiliensium species novae; ou histoire naturelle des espèces nouvelles de singes et de chauves-souris observées et recuellies pendant le voyage dans l'intérieur du Brésil... Munich, VIII+72p.

Recebido em 13.I.1998; aceito em 30.IV.1999, 